\title{
Adesão à prescrição médica em idosos de Porto Alegre, RS
}

\section{Medication adherence of elderly in Porto Alegre, RS}

\author{
Cristiane H offmeister Rocha ${ }^{1}$ \\ Ana Paula Sueiro de Oliveira ${ }^{1}$ \\ CarolineFerreira ${ }^{1}$ \\ FabianaTôrres Faggiani ${ }^{1}$ \\ GuilhermeSchroeter $^{1}$ \\ Antônio Carlos Araújo de Souza 2,3 \\ Geraldo Attilio DeCarli 1,2 \\ Fernanda Bueno $\mathrm{M}_{\text {orrone }}{ }^{1}$ \\ MariaCristina Werlang ${ }^{1}$
}

\footnotetext{
1FaculdadedeFarmácia, Pontifícia Universidade Católica do Rio Grande do Sul. Av. I piranga 6681, Prédio 12, Bloco A, Partenon. 90619-900 Porto Alegre RS.

crishrocha21@yahoo.com.br 2 Instituto de Geriatria e Gerontologia, PUCRS. ${ }^{3} \mathrm{H}$ ospital São Lucas, PUCRS.
}

Abstract Polipharmacy and medication non-adherence are problemsfaced frequently in the treatment of elderly patients. An exploratory cross-sectional study and quantitative approach were conducted to assess the frequency of treatment-adherence in elderly and how polipharmacy can affect adherence. Four hundred and sixty six elderly answered a questionnaire in Porto Alegre, RS in individual interviews. The adherence frequency found was $173(37.1 \%)$ and was higher among those, who use less medication. These results indicate the need for implementing educational programs for the elderly in order to help them to follow their drug therapy.

Key words Medication-adherence, Pharmacist care, Elderly
Resumo Polifarmácia e falta de adesão à prescrição médica são problemas freqüentes na terapêutica farmacológica de idosos que podem prejudicar o resultado do seu tratamento. Realizouse um estudo transversal exploratório e quantitativo em base populacional para levantar a freqüência de idosos aderentes e avaliar se a polifarmácia interfere na adesão. A pesquisa foi realizada com 466 idosos de Porto Alegre, RS. Os instrumentos foram aplicados por entrevista individual. A freqüência de idosos aderentes foi de $173(37,1 \%)$ e maior entre os que utilizavam menos fármacos. Os resultados encontrados sugerem a implementação de programas educacionais para auxiliar os idosos no seguimento à terapêutica farmacológica.

Palavras-chave Adesão à prescrição médica, Assi stência farmacêutica, Idosos 
Introdução

A partir da década de 70 , constata-se um crescimento considerável do contingente populacional de brasileiros com mais de 60 anos. Segundo dados do Consel ho Estadual do Idoso, atualmentehá cerca de 17 milhões de idosos no Brasil e prevê-se que no ano de 2030 esse número aumentará para 35 milhões ${ }^{1}$, sendo esse o segmento de maior crescimento populacional $2,3,5,5,7,8$.

A idade é uma variável preditora da terapia medicamentosa e seu efeito se produz mesmo antes dos 60 anos, pois a chance de usar algum tipo de fármaco aumenta desde a quarta década de vida ${ }^{9}$. Por causa da incidência de muitas patologias que acometem esta faixa etária, estes indivíduos tendem a ser maiores usuários de medicamentos. Segundo Flores et al., em Porto Alegre, $91 \%$ dos idosos fazem uso de al gum fármaco, sendo que $27 \%$ dos idosos entrevistados utilizavam cinco ou mais medicamentos ${ }^{6}$. As mudanças fisiológicas relacionadas ao envelhecimento podem alterar as propriedades farmacocinéticas efarmacodinâmicas no pacienteidoso ${ }^{2,5,9,10}$.

Além disso, o quadro de declínio cognitivo, as limitações físicas e as múltiplas doenças crônicas associadas podem afetar a sua habilidade de usar adequadamente os medicamentos. 0 conjunto de todos estes fatores pode comprometer o seu seguimento fidedigno em relação à terapêutica prescrita, ou seja, sua aderência à prescrição médi-

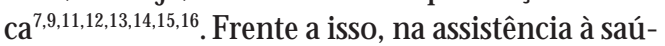
dedo idoso, percebe-seumaintensa relação entre o uso correto dos medicamentos pelos pacientes eas atividades exercidas pelo farmacêutico na prática da Atenção Farmacêutica ${ }^{6,10,17,18}$.

A partir do exposto acima, a presente pesquisa se propõe a investigar a freqüência de pacientes idosos aderentes à prescrição médica no município de Porto Alegre. Além disso, também objetiva avaliar se a polifarmácia interfere na adesão por estes pacientes. Para tanto, primeiramente será apresentada uma breve revisão acerca de aspectos relativos ao processo de envelhecimento e da adesão à prescrição médica por pessoas com mais de 60 anos.

\section{0 processo do envelhecimento}

0 envelhecimento do homem é um processo dinâmico e progressivo, no qual há alterações morfológicas, fisiológicas, bioquímicas e psicológicas, que determinam a perda progressiva da capacidade de adaptação do indivíduo ao meio ambiente, ocasionando maiores vulnerabilidade eincidência de processos patológicos, queterminam por levá-lo à morte ${ }^{2}$. As expectativas específicas de cada idade e a influência cultural também conceituam o processo deenvel hecimento ${ }^{2,4}$, o qual está intimamente ligado à manutenção da autonomia em relação ao desempenho das atividades diárias²,18,19.

A farmacologia para os idosos apresenta peculiaridades. As mudanças fisiológicas, determinadas pelo envelhecimento, levam a modificações nas propriedades farmacocinéticas e farmacodinâmicas dos medicamentos. Com a idade, diminui a massa muscular ea água corporal. 0 metabolismo hepático, os mecanismos homeostáticos, assim como a capacidade de filtração e de excreção renal, podem ficar comprometidos. Disso decorrem dificuldade deeliminação demetabólitos, acúmulo de substâncias tóxicas no organismo e a produção de reações adversas $5^{5,9,10}$.

Frente a estas alteraçõ̃es e, considerando a ele vação da freqüência dedoenças crônico-degenerativasqueacometeosidosos, o processo de envelhe cimento predispõe a um consumo aumentado de medicamentos ${ }^{6,7,9,18,20}$ e, conseqüentemente, àocorrência de problemas relacionados aos mesmos.

Dentre os problemas relacionados ao uso de medicamentos e que contribuem à não-adesão medicamentosa, encontram-se: automedicação, polifarmácia, interações farmacológicas e aparecimento de reações adversa5 $5^{5,2,21}$.

A polifarmacoterapia, conceituada como o uso concomitante de vários medicamentos, favorece sinergismos e antagonismos não desejados, descumprimento das prescrições dos fármacos clinicamente essenciais e gastos excedentes com os deuso supérfluo, contribuindo ànãoaderência medicamentosa?.

\section{Adesão à prescrição médica}

Diante de várias posições quanto à terminologia adequada para definir adesão ou aderência à prescrição médica, a Organização Mundial de Saúde a define como a magnitude com que o comportamento de um paciente coincide com o aconseIhamento do seu médico ${ }^{11,12,22,27,28}$. Os termos cumprimento, obediência, observância também são empregados na linguagem atual para definir o seguimento do paciente às instruções de seu médico $11,12,22,27,28$.

0 termo aderência ou adesão expressa compreensão e cooperação, subentendendo um comportamento ativo por parte do doenteesu- 
gerindo um envolvimento colaborativo no processo de planejamento eimplantação do seu tratamento ${ }^{11,12,28}$.

Ainda em termos de definição de nomenclatura, é necessário que se faça a distinção entre descontinuar e não-aderir ao tratamento. Descontinuar é cessar a administração do medicamento. Já a não-aderência pode permitir a continuação do tratamento, uma vez que o esquecimento de um determinado medicamento pode ser permanente ou temporário. Tanto a descontinuação do tratamento quanto a própria nãoaderência repercutem em um grande impacto para a saúde do paciente idoso, quando se considera o fármaco, a severidade da patologia e a sua comorbidade. Em determinadas situações, o que talvez ocorra com freqüência é que a incorreta aderência seja uma precursora da descontinuidade do tratamento 11,12,22,27,28.

\section{Fatores interferentes na adesão}

0 acometimento de reações adversas e as manifestações idiossincráticas podem interferir decisivamente na adesão medicamentosa. 0 medo do paciente em apresentar efeitos adversos dos medicamentos pode fazê-lo interromper o tratamento ${ }^{7,19,24}$.

0 tipo de enfermidade tratada também refletena aderência medicamentosa. Tratamentos crônicos ou de longa duração têm, em geral, menor adesão, visto que os esquemas terapêuticos exigem um grande empenho do paciente, que, em algumas circunstâncias, necessitam modificar seus hábitos devida para cumprir seu tratamento ${ }^{22,26,28}$.

A exposição a múltiplos medicamentos expõe o idoso a um tratamento mais complexo, exigindo maior atenção, memória e organização diante dos horários de administração dos fár$\operatorname{macos}^{5,6,18}$. Segundo Teixeira et al., as propriedades cognitivas encontram-se afetadas no paciente idoso, o que resulta em certa dificuldade para o seu entendimento e/ou para a recordação correta dos seus regimes terapêuticos prescritos ${ }^{27,24}$.

\section{M étodos deidentificação da adesão}

No que se refereà identificação do pacientenãoaderente, podem ser utilizados alguns recursos como: controle sérico dos fármacos, contagem de comprimidos, observações de aparecimento de reações adversas, avaliação das prescrições e questionários $\varsigma^{4,11,16,24,29}$.
O controle sérico dos fármacos na corrente sangüínea e/ou urina éum método mais preciso, não fica sujeito à honestidade do doente; no entanto, é o mais dispendioso e de difícil alcance ${ }^{11}$. As diferenças genéticas entre os indivíduos, segundo M idenceet al., podem interferir na análise da sua adesão ${ }^{11,21}$, já que elas refletem num comportamento distinto na farmacocinética dos medicamentos ${ }^{21}$.

A contagem de comprimidos, a percepção de reações adversas, a avaliação das prescrições e 0 questionamento se enquadram como medidas indiretas de adesão. Estes métodos são mais práticos, mas servem como um indicativo, não podendo ser considerados medidas precisas, já que dependem do relato correto do paciente. Neste sentido, não devem ser empregados isoladamente $^{4,5,11,24,26,27 \text {. }}$

A estimativa da aderência, por meio da contagem de comprimidos, se dá pela comparação entre a quantidade de medicamentos fornecidos na farmácia com a que o paciente leva na consulta 4,24,29. Em certos países, existem contadores de comprimidos; neste caso, cada vez que o paciente abre a embalagem e administra algum fármaco, esteéregistrado. No Brasil, são poucas as apresentações farmacêuticas que possuem estes marcadores, ficando restrito aos inaladores para afecções pulmonares e a alguns tipos de canetas de aplicação de insulina que registram as doses administradas. Além disso, pelo fato de não haver um sistema de atenção farmacêutica regular nas farmácias, as embalagens que não possuem este sistema não permitem a realização de tal controle $e^{16,24}$.

A resposta do paciente, por meio do relato el ou observação do aparecimento de reações adversas ou dos benefícios esperados da terapia, também pode ser indicativa da adesão ${ }^{11,19,24}$. A implantação deum plano de assistência farmacêutica ao paciente, no qual se proceda a um controle das quantidades de medicamentos fornecidas e administradas previamente, pode também identificar o comportamento do paciente em relação ao seguimento da terapia prescrita pelo seu médico. Porém, esta medida se encontra implementada em apenas alguns serviços farmacêuticos ${ }^{19,24}$.

0 método maisutilizado para detectar a falta de aderência é o questionamento direto ao paciente, devido a sua praticidade e baixo custo ${ }^{4}$. M orisky realizou um estudo durante três anos, para validar um questionário de levantamento da adesão, em pacientes hipertensos. 0 instrumento era composto por quatro perguntas fechadas, para as quais os pacientes respondiam sim ou não. A partir do escore obtido, resultava 
a seguinteclassificação: aderente, moderadamente aderente e não-aderente. Os resultados mostraram que $75 \%$ dos pacientes classificados como aderentes por meio do questionário possuíam seus níveis pressóricos dentro dos limites da normalidade ${ }^{11,12,24}$.

Num outro estudo, foram comparados os resultados de detecção de adesão por meio de diferentes técnicas, tais como: questionamento, contagem de comprimidos e avaliação de prescrição. As médias de freqüências de aderência encontradas pel os autores foram de $95,8 \pm 17,1 ; 74,0 \pm 41,5 \mathrm{e}$ $107,6 \pm 40,3(p<0,001)$, respectivamente.

Em suma, dependendo do estudo realizado, do grupo pesquisado edo método de detecção, as taxas de não-adesão podem variar de 13 a $93 \%{ }^{30}$.

\section{Metodologia}

\section{Desenho do estudo}

Desenvolveu-seum estudo transversal, exploratório, quantitativo e de base populacional, 0 qual fez parte do Projeto I dosos da Prefeitura do município de Porto Alegre, em parceria com o Instituto de Geriatria e Gerontologia da Pontifícia Universidade Católica do Rio Grande do Sul.

\section{Amostra}

A pesquisa foi realizada com 466 pessoas, com idade a partir de 60 anos e de ambos os sexos. 0 recrutamento dosidosos foi realizado a partir de dados fornecidos pela Prefeitura de Porto Alegre, conforme metodologia empregada no censo populacional do ano de2000. 0 contato foi realizado por telefone com apoio do Instituto de Geriatria e Gerontologia da PUCRS. Para participação desta pesquisa, foram considerados critérios de inclusão: não estar impossibilitado de se deslocar atéo local da entrevista efazer uso de medicamentos.

\section{Coleta de dados}

Os dados foram coletados entre janei ro esetembro de 2006 por meio de entrevistas individuais no ginásio de esportes da PUCRS. Para tanto, foram utilizados os seguintes instrumentos: ficha para levantamento de dados sociodemográficos, perfil farmacoterapêutico e um questionário auto-relatado para levantamento da aderência à terapêutica composto por quatro (04) perguntas fechadas, seguidas de uma pergunta aberta para 0 caso de seguimento à prescrição. Esta pergunta tinha por finalidade identificar o medicamento implicado.

Para este questionário, as respostas eram pontuadas em sim ou não, sendo atribuído o valor de zero para cada resposta afirmativa (em relação à adesão) e um (em relação à não-adesão) para as negativas. Escore zero indicava máxima adesão, de 1 a 2, moderada e de 3 a 4, baixa adesão. Considerando-se que, no presente estudo, apenas $11,3 \%$ dos pacientes apresentaram escore relativo à baixa adesão, utilizou-seo critério também adotado por outros autores: escore zero indicando adesão e a partir de 1, não-adesão ${ }^{31}$.

0 projeto foi aprovado pelo Comitê de Ética em Pesquisa da Pontifícia Universidade Católica do Rio Grande do Sul, conforme parecer número 0502935 .

\section{Análise estatística}

Os dados coletados foram tabulados e processados por meio do software SPSS (Statistical Package for Social Sciences) versão 11.5, por meio de estatísticas descritiva e analítica.

Os medicamentos relatados pelos pacientes foram classificados segundo a Anatomical-Therapeutical-Chemical Classification System (ATC).

Para avaliar a adesão de acordo com o sexo e o número de medicamentos utilizados, foi utilizado o teste estatístico do qui-quadrado, sendo considerado como significante $p<0,05$.

\section{Resultados}

A partir da análise dos dados, verificou-se que a amostra era composta em sua maioria por idosos do sexo feminino $356(69,1 \%)$. Dentre os indivíduos estudados, 270 (57,9\%) administravam de um a trêsfármacose 196 (42,1\%) administravam a partir de quatro medicamentos. 0 consumo médio de medicamentos por idoso foi de 3,3 $(\mathrm{DP}=2,62)$.

Em relação à classificação da adesão, 173 $(37,1 \%)$ dos idosos se auto-relataram aderentes e 293 (62,9\%), não-aderentes. As freqüências de pacientes aderentes por sexo encontram-se descritas na Tabela 1. Como pode ser observado, não foram encontradas diferenças significativas entre os sexos $(p>0,05)$.

Ao comparar-se a classificação da adesão segundo o número de medicamentos utilizados, a freqüência de pacientes foi maior entre os que utilizavam um menor número de medicamen- 
tos. Estes resultados foram estatisticamente significativos $\left(\chi^{2}=3,95 ; p=0,047\right)$, e encontram-se descritos na Tabela 2.

As respostas obtidas para cada pergunta da Escala de M orisky foram examinadas individualmente e encontram-se descritas na Tabela 3. De acordo com os dados ali descritos, verificase que a falta de adesão da maioria dos idosos se deve ao fato de se esquecerem de tomar seus medicamentos.

Em caso de resposta negativa às questões da Escala de Morisky, os pacientes foram questionados sobre os medicamentosimplicados. A classe terapêutica mais mencionada foi a dos medicamentos do aparelho cardiovascular, seguido pelos fármacos do sistema músculo esquelético. As freqüências encontradas para as quatro per-

Tabela 1. Freqüência de pacientes aderentes por sexo.

\begin{tabular}{lccccc}
\hline & \multicolumn{2}{c}{ Feminino } & \multicolumn{2}{c}{ Masculino } & \multicolumn{1}{c}{$\square$} \\
\cline { 2 - 4 } & $\mathrm{n}$ & $(\%)$ & $\mathrm{n}$ & $(\%)$ & \\
\hline Aderente & 120 & $(69,4)$ & 53 & $(31,2)$ & 0,274 \\
Não-aderente & 217 & $(74,1)$ & 76 & $(25,9)$ & 0,274 \\
\hline
\end{tabular}

= nível de significância calculado por meio do teste estatístico qui-quadrado.

Tabela 2. Freqüência de pacientes aderentes por número de medicamentos utilizados.

\begin{tabular}{lrrrr}
\hline & $\begin{array}{c}\text { De } 1 \text { a 3 } \\
\text { fármacos }\end{array}$ & \multicolumn{2}{c}{$\begin{array}{c}\text { A partir de } \\
\text { 4 fármacos }\end{array}$} \\
\cline { 2 - 5 } & $\mathrm{n}$ & $(\%)$ & $\mathrm{n}$ & $(\%)$ \\
\hline Aderente & 90 & $(52,0)$ & 83 & $(48,0)$ \\
Não-aderente & 180 & $(61,4)$ & 113 & $(38,6)$ \\
\hline
\end{tabular}

guntas foram de $10,9 \%, 9,7 \%, 7,6 \%$ e 7,4\%, respectivamente, para os fármacos do aparel ho cardiovascular e 5,2\%, 3,7\%, 6, $4 \%$ e 7,0\%, respectivamente, para aqueles pertencentes ao sistema do músculo esquelético.

\section{Discussão}

De acordo com os dados encontrados no presente estudo, verificou-se uma maior freqüência das mulheres no uso de medicamentos. Estere sultado é semel hante a achados de estudos prévios. Segundo Brand et al., em sua pesquisa, $67 \%$ dos idosos eram do sexo feminino, assim como no estudo deFlores et al. o gênero feminino prevalecia. Na pesquisa de Teixeira et al., apenas $23 \%$ dos idosos eram do sexo masculino. Este dado podeser justificado pela maior expectativa devida da mulher, em relação ao homem, no Brasil. A proteção cardiovascular dada pelos hormônios femininos, o menor consumo de tabaco eálcool, além da maior procura por assistência médica são hipóteses levantadas por al guns autores para explicar essa diferença populacional entre os sexos na terceira idade $e^{6,18,32}$.

Com relação à freqüência de adesão ao tratamento pelo sexo dos pacientes, no presente estudo, o sexo não interfere na adesão. Este dado encontrado contradiz com pesquisas prévias. Segundo Ribeiro et al.,os pacientes do sexo feminino abandonaram menos o tratamento farmacológico que os do sexo masculino, cujas freqüências observadas foram de $12 \%$ e $26 \%$, respectivamente. A maior taxa de pacientes aderentes do sexo feminino podeser atribuída à aten ção dada pelas mulheres quanto ao aparecimento de problemas de saúde, com conseqüente aumento na utilização dos serviços médicos ${ }^{6,18,35,36}$.

Tabela 3. Freqüência de idosos que responderam afirmativamente às questões da Escala de M orisky.

\begin{tabular}{lrr}
\hline \multicolumn{1}{c}{ Questões } & \multicolumn{2}{c}{ Sim } \\
\cline { 2 - 3 } & \multicolumn{1}{c}{$\mathrm{n}$} & $(\%)$ \\
\hline O(A) sr(a) alguma vez se esquece de tomar os seus remédios? & 98 & $(33,4)$ \\
O(A) sr(a) é descuidado com os horários de tomar os seus remédios? & 73,2 & $(25)$ \\
Q uando o(a) sr(a) está sesentindo melhor, às vezes pára detomar os seus remédios? & 68,2 & $(23,3)$ \\
Algumas vezes, se o(a) sr(a) se sentiu mal, aumentou a quantidade de remédio a & 77,4 & $(26,4)$ \\
ser tomada? & & \\
\hline
\end{tabular}


A quantidade média de medicamentos observada no presente estudo assemelha-se com estudos já realizados. Segundo Flores et al., a média de fármacos por pessoa foi de 3,2 ( $D P=2,5)$, assim como aquela de 3,24 encontrada no estudo de Rozenfeld ${ }^{9}$. Na pesquisa de Blanski, a média de consumo de medicamentos por idosos foi de 4,0 fármacos. Segundo Teixeira et al., mais de $80 \%$ dos idosos tomam no mínimo um medicamento diariamente eapenas $4 \%$ a $10 \%$ dessa faixa etária não faz uso de qualquer fármaco ${ }^{7}$. Segundo Rozenfeld, o fácil acesso a medicamentos e a baixa freqüência do uso de recursos não farmacológicos para o manejo de problemas médicos contribuem para este perfil ${ }^{9}$.

Em relação à classe terapêutica cardiovascular ser a mais utilizada pelos idosos, neste estudo, os resultados foram similares aos já descritos na literatura. Segundo Blanski et al., 53,3\% dos idosos faziam uso de anti-hipertensivos; já na pesquisa de Filho et al., as categorias terapêuticas mais freqüentes incluíram medicamentos com ação no sistema cardiovascular (29,3\%), sistema nervoso central $(13,5 \%)$, metabolismo $(12,6 \%)$ e trato digestivo (12,3\%). Também no estudo de Flores et al. realizado na região Sul do Brasil, os medicamentos cardiovasculares foram os mais utilizados. Tal perfil de consumo pode ser justificado pelo fato das doenças cardiovasculares, que lideram as causas de morbi-mortalidade em indivíduos com idade acima de 65 anos, acarretarem maior consumo de fármacos.

Com relação à polifarmácia, no presente estudo, o uso de vários medicamentos é um fator prejudicial na adesão, resultado que se assemeIha a estudos prévios. Segundo Blanski et al., o motivo primeiro que interfere na adesão ao tratamento medicamentoso foi o uso concomitante de vários medicamentos, o que foi verificado em $15(33,3 \%)$ pacientes. Conforme Flores et al., a polifarmácia aumenta com a progressão da idade, fenômeno que pode ser explicado por uma série de fatores, incluindo aumento da morbidade; além disso, estima-se que $23 \%$ da população brasileira consomem $60 \%$ da produção nacional de medicamentos, principal menteas pessoas acima de 60 anos$^{9}$. No estudo de Rozenfeld, verificam-se indícios de aumento de "multiuso" em idosos. Nesta faixa etária, as doenças são mais graves, o uso de serviços médicos é mais intenso ehá dificuldade dediscutir os complexos problemas, sendo assim medicalizados 9 .

Segundo Gusmão, para avaliar as razões de não-adesão citados pelos pacientes, $30 \%$ dos indivíduos não administraram o seu medicamen- to por esquecimento, $11 \%$ optaram por uma dose menor do que a prescrita, $9 \%$ alegaram falta de informações e 7\% mencionaram fatores emocionais. Esse mesmo estudo mostrou que $27 \%$ dos indivíduos avaliados não souberam dar uma razão para a baixa adesão ao tratamento ${ }^{28}$. Já no estudo realizado por Rozenfeld ${ }^{9}$ com pacientes hipertensos, $89 \%$ dos indivíduos referiram baixa adesão devido ao alto custo do tratamento prescrito, $67 \%$ pelo elevado número de doses a serem administradas diariamente e $54 \%$ pelos efeitos indesejáveis causados pelos medicamento ${ }^{18}$. Estes dados reforçam os resultados encontrados. No presenteestudo, dentreos pacientes entrevistados, 33,4\% deixaram de seguir sua prescrição pelo esquecimento, $25 \%$ por descuido com os horários de administração, 23,3\% interrompiam o seu tratamento pela melhora dos sintomas e 26,4\% aumentavam a quantidade de fármacos pela piora dos sintomas.

A não conscientização por parte do paciente na questão do seguimento da terapia é um dos problemas comumente verificados entre os ido$\operatorname{sos}^{4,5,9}$. Este fato pode ser observado no presente estudo, quando $23,3 \%$ dos idosos afirmaram que paravam de administrar seus medicamentos quando se sentiam melhor, e também quando $26,4 \%$ dos entrevistados mencionaram que aumentavam a dose prescrita quando sentiam piora dos sintomas que estavam sendo tratados. Em ambas as situações de não-adesão, os fármacos mais mencionados foram os do sistema cardiovascular.

A ausência de sintomas em algumas fases do processo de adoecimento leva, em certas patologias, os pacientes a não aderirem aos seus tratamentos ${ }^{26}$. Além disso, o aumento da dose dos seus fármacos pode trazer sérios riscos a sua saúde, principalmente quando os medicamentos mais implicados nesta questão foram os cardiovasculares. Sendo assim, a orientação dos profissionais de saúde aos pacientes torna-se fundamental na adesão ao tratamento.

\section{Consideraçõesfinais}

O número de publicações acerca dos idosos aumentou exponencialmente nos últimos anos, acompanhando as modificações demográficas que revelam um aumento progressivo dessa faixa etária em todo o mundo, inclusive no Brasil ${ }^{17}$. Por outro lado, surpreende o reduzido número de estudos encontrados dado a relevância do tema. 
Os dados do presente estudo indicam que a maioria da população idosa de Porto Alegre não adereà prescrição médica. Entretanto, foi encontrada uma maior freqüência de pacientes aderentes entre aqueles que utilizam um menor número de medicamentos. De acordo com o instrumento utilizado para investigar a adesão, 0 motivo mais freqüentemente mencionado pelos pacientes foi o próprio esquecimento, seguido da prática de aumentar a dose dos medicamentos prescritos ao sentir piora dos sintomas.

A partir disso, o envolvimento de toda equipe de saúde na condução de programas que auxiliem o idoso no seguimento da terapia medicamentosa torna-se fundamental tanto paraa identificação de possíveis problemas que possam estar interferindo na sua adesão à prescrição como também para a conscientização acerca de uma farmacoterapia responsável. N este contexto, é importante que os profissionais envolvidos, médicos, farmacêuticos, enfermeiros, desenvolvam um relacionamento colaborador com o paciente, para que o idoso se sinta à vontade para relatar com fidedignidade sua conduta.

A condução de tais programas inclui a definição de estratégias para a prescrição e para a orientação ao paciente. A primeira envolveavaliar a real necessidade de prescrição, procurando-se reduzir ao máximo a complexidade do regime posológico, e, quando possível, utilizar apresen- tações com associações medicamentosas. Um outro aspecto a considerar é a identificação de multiuso de medicamentos pelo idoso. Paratanto, o médico pode contar com a colaboração da equipe de saúde no sentido de averiguar se o pacienteé usuário de polifarmácia.

Já no que se refere ao aspecto da orientação, o profissional farmacêutico pode contribuir informando aos pacientes sobre sua patologia e os medicamentos a serem administrados. A orientação, que pode ser tanto verbal como por escrito, inclui instruções sobre o modo correto deusar, possíveis efeitos adversos, atitude a tomar no caso de esquecimento de doses, alertas quanto aos ris$\cos$ da automedicação e da interrupção prévia do tratamento. Caso o farmacêutico detecte algum problema com a terapia farmacológica prescrita, ou mesmo de dificuldade de adesão, deve entrar em contato com o médico para discutirem uma possível intervenção.

A falta de adesão à prescrição médica, além de incidir em custos desnecessários, prejudica a resposta do paciente frente ao tratamento proposto, podendo, muitas vezes, ser fatal. Neste contexto, por meio do estabelecimento de medidas interventoras, reunindo-se os esforços da equipe de saúde, tem-se o intuito de conscientizar o paciente idoso quanto à importância da sua adesão à prescrição, contribuindo-se assim com sua qualidade de vida.

\section{Colaboradores}

CH Rocha realizou a revisão bibliográfica, redigiu 0 artigo, colaborou com as entrevistas e procedeu a tabulação dos dados. FT Faggiani, G Schroeter, AP Sueiro e C Ferreira participaram das entrevistas e da tabulação dos dados. ACA de Souza coordenou o Projeto Idosos de Porto Alegre. FB Morrone conduziu a investigação e revisou o texto. M C Werlang orientou a pesquisa, fazendo a revisão final do texto.

\section{Agradecimentos}

$\mathrm{O}$ estudo foi financiado pelo Conselho $\mathrm{N}$ acional de Pesquisa (CNPq) e Prefeitura Municipal de Porto Alegre, RS. Contou com o apoio logístico do Instituto de Geriatria e Gerontologia da PUCRS, H ospital São Lucas da PUCRS e Faculdade de Farmácia da PUCRS. Os autores agradecem a colaboração de Ana Lígia Bender, Graciane Radaelli, J oão Paulo Cassol, Liamara Andrade, Flávia Valladão Thiesen, Luiz Fernando Rodrigues, M ariele Motter, Michele Ruano, Rafaela Nascimento , Sandro Luiz Pacheco, Tamara Trombetta, Terezinha M unhoz, Thiago M ilech de Assunção e Virgínia M ingueli Schmitt pela participação na etapa de coleta de dados. 


\section{Referências}

1. Conselho Estadual do Idoso. Os idosos do Rio Grande do Sul: Estudo multidimensional de suas condições de vida. Porto Alegre: Governo do Estado do Rio Grande do Sul-CEI; 2006.

2. Filho ETC. Fisiologia do envelhecimento. In: Papaléo NM, organizador. Gerontologia: a velhice e o envelhecimento em visão globalizada. São Paulo: Atheneu; 1996. p. 60-70.

3. Paschoal SM P. Epidemiologia do envelhecimento. In: Papaléo NM, organizador. Gerontologia: a velhice e 0 envelhecimento em visão globalizada. São Paulo: Atheneu; 1996. p. 26-43.

4. Diaz RB. Adesão ao tratamento medicamentoso em idosos. In: Papaléo NM, organizador. Gerontologia: a velhice e 0 envelhecimento em visão globalizada. São Paulo: Atheneu; 1996. p. 230-241.

5. Gallo JJ, Whitehead JB, Rabins PV, M urphy JB. Reichel assistência ao idoso: aspectos clínicos do envelhecimento: Rio de Janeiro: Guanabara Koogan; 2001.

6. Flores LM, M engue SS. U so de medicamentos por idosos em região do sul do Brasil. Rev Saúde Pública 2005; 39(6):924-929.

7. Teixeira JJ, Lefèvre $F$. A prescrição medicamentosa sob a ótica do paciente idoso. Rev. Saúde Pública 2001; 35(2):207-213.

8. Nóbrega OT, Karnikowski M GO. A terapia medicamentosa no idoso: cuidados na medicação. Cad Saúde Coletiva 2005; 10(2):309-313.

9. Rozenfeld S. Prevalência, fatores associados e mau uso de medicamentos entre os idosos: uma revisão. Rev. Saúde Pública 2003; 19(3):717-724.

10. Coelho FJM, M arcopito LF, Castelo A. Perfil de utilização de medicamentos por idosos em área urbana do Nordeste do Brasil. Rev. Saúde Pública 2004; 38(4):557-564.

11. Midence $K$, M yers LB. Adherence to treatment in medical conditions. London: Harwood Academic Publishers; 1998.

12. Morisky DE, Green LW, Levine DM. Concurrent and predictive validity of a self-reported measure of medication adherence. M edical Care 1986; 24(1):67-74.

13. Vitolins MZ, Rand CS, Rapp SR, Ribisl PM, Sevick MA. M easuring adherence to behavioral and medical interventions. Clin Control Trials 2000; 21(5):188S-194S.

14. Brawley LR, Culos-Reed SN. Studying adherence to therapeutic regimens: overview, theories, recommendations. Clin Control Trials 2000; 21(5):156S-163S.

15. Chen YF, Dewey ME, Avery AJ. Self-report medication use for older people in England and Wales. Journal of Clinical Pharmacy and Therapeutics 2001; 26:129-140.

16. Schlenk, EA, Dunbar JJ, Engberg S. M edication nonadhrence among older adults: a review of strategies and interventions for improvement. Journal of Gerontological Nursing 2004; 30(7):33-43.

17. Romano-Lieber NS, Teixeira JJV, Farhat FCLG, Ribeiro $E$, Crozatti Márcia TL, Oliveira GSA. Revisão dos estudos de intervenção do farmacêutico no uso de medicamentos por pacientes idosos. Rev. Saúde Pública 2002; 18(6):1499-1507.

18. Blanski CR, Lenardt MHA. Compreensão da terapêutica medicamentosa pelo idoso. Nursing Journal of Rio Grande do Sul 2005; 26(2):137-281.

19. M arcolongo R, Ribeiro E, Storpirtis S. Novas diretrizes para assistência farmacêutica hospitalar: atenção farmacêutica/farmácia clínica. In: Gomes MJVM, Reis AM M, organizadores. Ciências farmacêuticas uma abordagem em farmácia hospitalar. São Paulo: Atheneu; 2001. p. 521-530.
20. Guerra Jr AA, Acúrcio FA, Gomes CAP, Miralles M, Girardi SN, Werneck GAF, Carvalho CL. Disponibilidade de medicamentos essenciais em duas regiões de M inas Gerais -Brasil. Rev Panam de Salud Pública 2004; 15(3):168-175.

21. Belon JP. Consejos en la farmacia. Barcelona: Masson; 1999.p. 3-16.

22. Leite SN, Vasconcellos M PC. Adesão à terapêutica medicamentosa: elementos para a discussão de conceitos e pressupostos adotados na literatura. Cad Saúde Col 2003; 8(3):775-782.

23. Lynch T. Medication costs as a primary cause of nonadherence in the elderly. Consult Pharm 2006; 21(2):143-146.

24. MacLaughlin EJ, Raehl CL, Treadway AK, Sterling $T L$, Zoller DP, Bond CA. Assessing medication adherence in the elderly: which tools to use in clinical practice? Drug Aging 2005; 22(3):231-55.

25. M ojtabai R, Olfson M. M edication costs adherence, and health outcomes among M edicare beneficiaries. Health Aff (M illwood) 2003; 22(4):220-229.

26. Leite SN. Adesão à terapêutica medicamentosa: 0 que o farmacêutico tem a ver com isso? In: Cordeiro BC, Leite SN, organizadores. Farmacêutico na atenção à saúde. Itajaí: Univali; 2005. p. 75-90.

27. M étry JM. M easuring compliance in clinical trials and ambulatory care. In: JM M étry, Meyer UA, editors. Drug regimen compliance. New York: Wiley; 1999.

28. Gusmão JL, Mion Jr. D. Adesão ao tratamento: conceitos. Rev Bras Hipert 2006; 13(1):23-25.

29. Grymonpre RE, Didur CD, M ontgomery PR, Sitar DS. Pill count, self-report, and pharmacy claims data to measure medication adherence in the elderly. The Ann Pharm 1998; 32(7):749-754.

30. Bond WS, Hussar DA. Detection methods and strate gies for improving medication compliance. American Journal of Hospital Pharmacy 1991; 48(9):1978-1988.

31. Ramalhinho I, Cabrita J. Avaliação do grau de adesão à terapêutica antihipertensiva pelo método da contagem de medicamentos. Revista Portuguesa de Saúde Pública 1998;16:5-12.

32. Jeckel Neto E, Cunha GL. Teorias biológicas do envelhecimento. In: Vianna EFM, Py L, N eri AL, Cançado FAX, Gorzoni ML, Rocha SM, organizadores. Tratado de geriatria e gerontologia. Rio de Janeiro: Guanabara Koogan; 2002. p. 13-19.

33. Raehl CL, Bond CA, Woods TJ, Patry RA, Sleeper RB. Screening tests for intended medication adherence among the elderly. Ann Pharm 2006; 40(5):888-893.

34. M orley PC, Cipolle RJ, Strand LM. Pharmaceutical Care Practice: The Clinician's Guide 2ªed. New York: McGraw-Hill Medical; 2004.

35. Ribeiro SA, Amado VM, Camelier AA, Fernandes M M A, Schenkman S. Estudo caso-controle de indicadores de abandono em doentes com tuberculose. Jornal de Pneumologia 2000; 26(6):291-296.

36. Issa JS, Forti N, Giannini SD, Diament J. Intervenção sobre tabagismo realizada por cardiologista em rotina ambulatorial. Arch Bras Cardiol 1998; 70(4):271-274.

Artigo apresentado em 08/01/2006

Aprovado em 16/01/2007

Versão final apresentada em 15/02/2007 